\title{
COMPOSICIÓN Y ESTRUCTURA DE LA COMUNIDAD DE OCTOCORALES EN LOS BAJOS DE SANCHO PARDO, ARRECIFE DE LOS COLORADOS, CUBA
}

\section{COMPOSITION AND STRUCTURE OF THE OCTOCORAL COMMUNITY IN BAJOS DE SANCHO PARDO, LOS COLORADOS REEF, CUBA}

\author{
Alejandro Pérez-Angulo ${ }^{1 *}$ y Daril de la Nuez Hernández ${ }^{2}$
}

\begin{abstract}
RESUMEN
Se estudiaron la composición y estructura de las comunidades de octocorales en cinco sitios localizados en los Bajos de Sancho Pardo (arrecife de Los Colorados (NW Cuba)) en agosto del 2010. Se empleó como unidad de muestreo un marco cuadrado de un metro de lado. En total se estudiaron 11 zonas del arrecife frontal, dos zonas traseras y una cresta. Se identificaron 34 especies de gorgonias de 2687 colonias. En el arrecife frontal, Pseudopterogorgia americana y Pseudopterogorgia elisabethae fueron las especies dominantes, mientras que en la zona trasera lo hicieron Briareum asbestinum y Pseudopterogorgia bipinnata. En la cresta arrecifal predominaron Gorgonia ventalina y Plexaura homomalla. Se encontraron diferencias significativas $(P=0.05)$ para la densidad de gorgonias entre localidades del arrecife frontal. Los resultados de los índices de heterogeneidad y de equitatividad permiten inferir que un $79 \%$ de las localidades se correspondieron con ambientes favorables o muy favorables y el $64 \%$ indicaron ambientes constantes o casi constantes. En el 79\% de las localidades se infirió una tensión hidrodinámica baja y en el resto resultó entre moderada y alta en zonas someras de la cresta y el arrecife frontal. El estado general de la comunidad de octocorales fue bueno, según los valores de densidad y abundancia de especies registrados, aunque se evidencia que en las zonas al oeste hay un mayor cubrimiento por macroalgas carnosas, que puede constituir un factor que limita el reclutamiento.
\end{abstract}

Palabras claves: Octocorales, arrecifes coralinos, indicadores ecológicos, Los Colorados, Cuba.

\begin{abstract}
The composition and structure of octocoral communities was studied in five locations in Bajos de Sancho Pardo (Los Colorados reefs (NW Cuba)) in August 2010. Quadrants measuring one square meter were used as sampling units. A total of 11 fore reefs, two rear zones and one reef crest were studied. Thirty-four species of gorgonians were identified in 2687 colonies. Predominant species included Pseudopterogorgia americana and Pseudopterogorgia elisabethae in the fore reef zone, Briareum asbestinum and Pseudopterogorgia bipinnata in the rear reef, and Gorgonia ventalina and Plexaura homomalla in the reef crest. Significant differences $(P=0.05)$ were found in the density of gorgonians between fore reef stations. Based on the results of the heterogeneity and evenness indexes, it can be inferred that $79 \%$ of the stations were consistent with favorable or very favorable environments and $64 \%$ with constant or nearly constant environments. Hydrodynamic stress was low in $79 \%$ of the stations, while it was moderate to high in shallow areas of the crest and fore reef. The overall condition of the octocoral community was good according to recorded values of species density and abundance, although it is evident that in the western areas there is greater coverage by fleshy macroalgae, which may be a limiting factor for growth.
\end{abstract}

Keywords: Octocorals, coral reefs, ecological indicators, Los Colorados, Cuba.

1, 2 Acuario Nacional de Cuba, Avenida 1ra. y 60, Playa, CP 11300, Ciudad Habana, Cuba. *alejandrop@acuarionacional.cu

Recibido 26-IV-2011

Aceptado 01-IX-2011

DOI: http://dx.doi.org/10.15359/revmar.3.10

Rev. Mar. Cost. ISSN 1659-455X. Vol. 3: 127-138, Diciembre 2011. 


\section{INTRODUCCIÓN}

En la actualidad, los ecosistemas marinos han sido influenciados por el efecto directo del cambio climático que incluye: el calentamiento del océano, su acidificación, la elevación del nivel del mar, cambios en los patrones de circulación e incremento en la severidad de las tormentas, entre otros (Keller et al. 2009; Schutte et al. 2010). Esto ha provocado que en los últimos años se hayan incrementado los estudios sobre los arrecifes coralinos, ecosistemas que tienen una gran importancia como reserva de extraordinaria diversidad, que brindan refugio, alimento y sustrato a numerosas especies de interés comercial o farmacológico (Vega et al. 2002).

Los octocorales son muy resistentes al fenómeno del blanqueamiento o la expulsión de sus simbiontes (Lasker, 2003; Drohan et al. 2005), fenómeno que afecta mucho a los arrecifes de coral y su incremento parece estar vinculado directamente con el cambio climático. Por ejemplo, en los arrecifes colombianos, a pesar de un declive de $20-30 \%$ en la cobertura del coral, los octocorales parecen crecer con éxito (Cadena y Sánchez, 2010). Por otra parte, Schutte et al. (2010) sugieren que la pérdida de los corales está también asociada con un incremento en la abundancia de otros taxones, tales como, esponjas, gorgonias y macroalgas.

Los octocorales normalmente son dominantes en número y biomasa entre los invertebrados bentónicos que componen el arrecife de coral en Cuba. Además, este es uno de los grupos focales utilizado como bioindicador de perturbaciones en el ambiente marino en dependencia de la abundancia, escasez o ausencia de determinadas especies en un sitio dado (Hernández y Alcolado, 2007; Hernández-Muñoz et al. 2008; Alcolado et al. 2008). Por ello, a partir de los resultados que se obtengan se podría inferir también el comportamiento ambiental bajo las condiciones actuales que refleja un determinado sitio.

El archipiélago de Los Colorados, al noroeste de Cuba, es una zona de arrecifes bien preservada, debido a lo distante que está de tierra y a la baja incidencia del hombre sobre este. Dicho lugar se encuentra cercano a áreas del océano profundo propuestas para la iniciación de la exploración petrolera desde mediados del 2009, lo que implica riesgos de posibles descargas o derrames accidentales de petróleo en el futuro (González-Díaz et al. 2010). Este riesgo se hace más evidente si se tiene en cuenta que por aquí pasan con frecuencia ciclones tropicales (seis de estos fenómenos meteorológicos se han registrado entre los años 2000 y 2005).

La información existente sobre las comunidades de octocorales de Sancho Pardo es muy escasa y se recoge en el trabajo de González-Díaz et al. (2010). Para el futuro manejo y conservación de esta área marina es necesario realizar un estudio sobre la composición y estructura de la comunidad de octocorales teniendo en cuenta su variabilidad espacial a lo largo de este arrecife. Ello permitiría establecer bases de referencia para futuras investigaciones, partiendo del empleo de este grupo del bentos como indicadores de la salud de este ecosistema.

\section{MATERIALES Y MÉTODOS}

\section{Área de estudio}

El estudio se realizó en un lugar conocido como los Bajos de Sancho Pardo, en el arrecife de Los Colorados, situado a lo largo de la región noroeste de la provincia de Pinar del Río, Cuba (Fig. 1). Este arrecife se localiza muy lejos de cualquier centro 
urbano (40 km) (González-Díaz et al. 2010) y los signos de contaminación no son evidentes (González-Sansón et al. 2009).

En invierno en los Bajos de Sancho Pardo impactan con frecuencia los frentes fríos, que provocan fuertes marejadas y vientos de región norte y nordeste. Además, en los meses de verano, es frecuente el impacto de ciclones tropicales, que inciden directamente sobre este arrecife (Alcolado et al. 2003). Los vientos alisios del nordeste dominan durante gran parte del año, y su efecto es menor hacia la porción oeste de los arrecifes que están más resguardados, porque su posición es más a sotavento en el borde de la plataforma.

Esta zona geográfica se caracteriza por la presencia de áreas conocidas como "bajos", con profundidades entre 1 y 3 $\mathrm{m}$. Las crestas se distribuyen a manera de parches, con predominio de Acropora palmata. Las zonas traseras se encuentran más resguardadas del impacto directo de las marejadas, y se localizaban continuamente al sur y oeste de los bajos. La profundidad en estas oscila entre cuatro y cinco metros y aquí dominan los "arrecifes de parches" y el sustrato

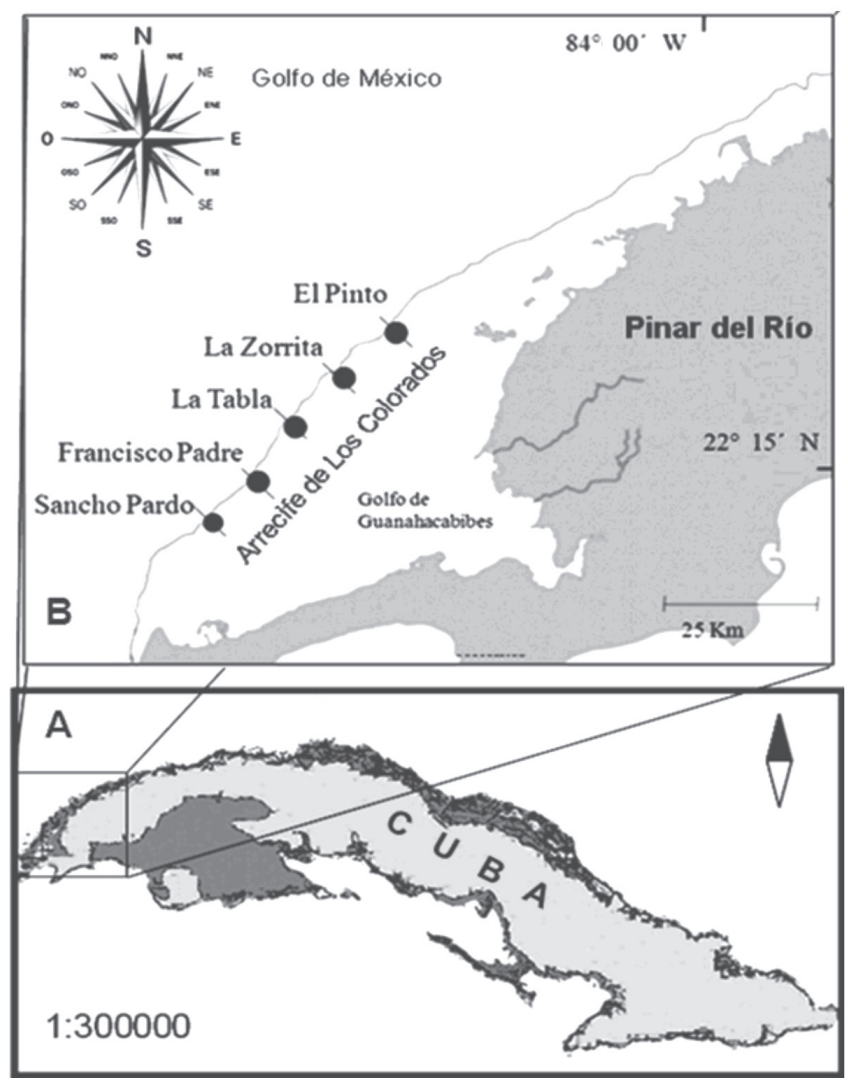

Fig. 1. Localización del área de estudio en la región noroeste de Cuba (A). Ubicación geográfica de los sitios de muestreo en el arrecife de Los Colorados (B)

Fig. 1. Location of study area in the northwestern region of Cuba (A). Geographic location of sampling sites in Los Colorados reef (B) 
es fundamentalmente coralino y en ocasiones se alterna con parches de arena.

Los arrecifes frontales presentan macizos y canales que incrementan su complejidad y altura con la profundidad. Este lugar estuvo dominado por corales, gorgonias y algas carnosas de los géneros Dictyota, Lobophora, Sargassum y Microdictyon. Los erizos negros Diadema antillarum estuvieron prácticamente ausentes.

\section{Metodología y procesamiento de datos}

La toma de muestras se hizo en agosto del 2010. Se establecieron cinco puntos ( $\mathrm{SP}=$ Sancho Pardo, $\mathrm{FP}=$ Francisco Padre, $\mathrm{LT}=\mathrm{La}$ Tabla, $\mathrm{LZ}=\mathrm{La}$ Zorrita, EP $=\mathrm{El}$ Pinto) separados aproximadamente $10 \mathrm{~km}$ entre sí, los cuales fueron georreferenciados (Fig. 1 y Cuadro 1). En cada punto se trabajaron los 15 y $10 \mathrm{~m}$ de profundidad y 5 $\mathrm{m}$ en La Tabla (zona ecológica del arrecife frontal), mientras que se trabajaron dos zonas traseras y una cresta (Cuadro 1).

Se empleó un marco cuadrado de un metro de lado, el que fue colocado aleatoriamente sobre el fondo en cada perfil batimétrico (Weinberg, 1981). En cada localidad se tomaron los datos de 30 marcos y las colonias se contaron e identificaron in situ; aquellas de clasificación dudosa se extrajeron para su posterior identificación a partir de los criterios de Bayer (1961) y Sánchez y Wirshing (2005). Se calculó la densidad $\left(\right.$ colonias $\left./ \mathrm{m}^{2}\right)$.

Para determinar si el tamaño de la muestra fue representativo se confeccionaron las curvas acumulativas para el número de especies (S) contra el número de individuos $(\mathrm{N})$. Se tomó como criterio la existencia de una tendencia asintótica en los valores de riqueza.

Se empleó el programa PRIMER 5.0 para calcular los índices ecológicos de Heterogeneidad (H') de Shannon y Weaver
(1949) y de Equitatividad (J') de Pielou (1966), utilizando los logaritmos neperianos, así como el número de especies para cada localidad. Se infirió el grado de favorabilidad y constancia ambiental para los octocorales a partir de la interpretación de H' y J' propuesta por Hernández y Alcolado (2007).

Para inferir el grado de agitación del agua en cada localidad se empleó el Índice de Tensión Hidrodinámica (ITH) propuesto por Alcolado (1981). Este se basa en calcular la suma de los porcentajes de las especies de octocorales que resisten este tensor. Hernández y Alcolado (2007) utilizan este índice dividiéndolo en las siguientes categorías: $0-20 \%=$ muy bajo, $21 \%-40 \%=$ bajo, $41 \%-60 \%$ $=$ moderado, $61 \%-80 \%=$ alto y de $81 \%$ $100 \%=$ muy alto.

Se realizó un análisis de varianza no paramétrico (ANOVA, de Kruskal-Wallis) por localidades, para conocer la existencia de diferencias significativas en cuanto a la densidad media. Se aplicó la prueba no paramétrica de comparación por rangos del programa Statistica 6.0 para establecer entre qué pares de medias se encontraban las diferencias significativas $(P=0.05)$.

\section{RESULTADOS}

En la zona de los Bajos de Sancho Pardo se contaron e identificaron 2687 colonias de octocorales, pertenecientes a cuatro familias, 11 géneros y 34 especies, y el número por localidades osciló entre 8 y 23. De ellas, Pseudopterogorgia americana (Gmelin, 1791) estuvo presente en todas las localidades y fue dominante principalmente en las zonas del arrecife frontal. Para esta misma zona ecológica, Pseudopterogorgia elisabethae (Bayer, 1961) dominó en Sancho Pardo, Francisco Padre y La Tabla. En la zona trasera, la 
Cuadro 1. Ubicación geográfica y zona ecológica de las localidades estudiadas en los Bajos de Sancho Pardo. Est. = estación, Prof. = profundidad, SP = Sancho Pardo, FP = Francisco Padre, LT $=$ La Tabla, $\mathrm{LZ}=$ La Zorrita, EP $=$ El Pinto

Table 1. Location and ecological zones of the sampling sites in the Bajos de Sancho Pardo. Loc. $=$ locality estation, Prof. $=$ depth, $\mathrm{SP}=$ Sancho Pardo, FP $=$ Francisco Padre, $\mathrm{LT}=\mathrm{La}$ Tabla, $\mathrm{LZ}=\mathrm{La}$ Zorrita and EP $=\mathrm{El}$ Pinto

\begin{tabular}{|c|c|c|}
\hline Loc. (Prof. (m)) & Coordenadas geográficas & Zonas ecológicas \\
\hline $\mathrm{SP}(15 \mathrm{~m})$ & $22^{\circ} 69^{\prime} 86^{\prime \prime} \mathrm{N}$ y $84^{\circ} 46^{\prime} 37^{\prime \prime} \mathrm{W}$ & arrecife frontal \\
\hline $\mathrm{SP}(10 \mathrm{~m})$ & $22^{\circ} 69^{\prime} 86^{\prime \prime} \mathrm{N}$ y $84^{\circ} 46^{\prime} 37^{\prime \prime} \mathrm{W}$ & arrecife frontal \\
\hline $\mathrm{SP}(5 \mathrm{~m})$ & $22^{\circ} 69^{\prime} 86^{\prime \prime} \mathrm{N}$ y $84^{\circ} 46^{\prime} 37^{\prime \prime} \mathrm{W}$ & zona trasera \\
\hline $\mathrm{FP}(15 \mathrm{~m})$ & $22^{\circ} 14^{\prime} 69^{\prime \prime} \mathrm{N}$ y $84^{\circ} 43^{\prime} 93^{\prime \prime} \mathrm{W}$ & arrecife frontal \\
\hline $\mathrm{FP}(10 \mathrm{~m})$ & $22^{\circ} 14^{\prime} 69^{\prime \prime} \mathrm{N}$ y $84^{\circ} 43^{\prime} 93^{\prime \prime} \mathrm{W}$ & arrecife frontal \\
\hline $\mathrm{LT}(15 \mathrm{~m})$ & $22^{\circ} 19^{\prime} 65^{\prime \prime} \mathrm{N}$ y $84^{\circ} 39^{\prime} 20^{\prime \prime} \mathrm{W}$ & arrecife frontal \\
\hline $\mathrm{LT}(10 \mathrm{~m})$ & $22^{\circ} 19^{\prime} 65^{\prime \prime} \mathrm{N}$ y $84^{\circ} 39^{\prime} 20^{\prime \prime} \mathrm{W}$ & arrecife frontal \\
\hline $\mathrm{LT}(1 \mathrm{~m})$ & $22^{\circ} 19^{\prime} 65^{\prime \prime} \mathrm{N}$ y $84^{\circ} 39^{\prime} 19^{\prime \prime} \mathrm{W}$ & cresta \\
\hline $\mathrm{LT}(5 \mathrm{~m})$ & $22^{\circ} 19^{\prime} 65^{\prime \prime} \mathrm{N}$ y $84^{\circ} 39^{\prime} 19^{\prime \prime} \mathrm{W}$ & zona trasera \\
\hline $\mathrm{LZ}(15 \mathrm{~m})$ & $22^{\circ} 23^{\prime} 03^{\prime \prime} \mathrm{N}$ y $84^{\circ} 37^{\prime} 21^{\prime \prime} \mathrm{W}$ & arrecife frontal \\
\hline $\mathrm{LZ}(10 \mathrm{~m})$ & $22^{\circ} 23^{\prime} 00^{\prime \prime} \mathrm{N}$ y $84^{\circ} 36^{\prime} 96^{\prime \prime} \mathrm{W}$ & arrecife frontal \\
\hline $\mathrm{LZ}(5 \mathrm{~m})$ & $22^{\circ} 22^{\prime} 65^{\prime \prime} \mathrm{N}$ y $84^{\circ} 35^{\prime} 83^{\prime \prime} \mathrm{W}$ & arrecife frontal \\
\hline $\mathrm{EP}(15 \mathrm{~m})$ & $22^{\circ} 26^{\prime} 84^{\prime \prime} \mathrm{N}$ y $84^{\circ} 31^{\prime} 19^{\prime \prime} \mathrm{W}$ & arrecife frontal \\
\hline $\mathrm{EP}(10 \mathrm{~m})$ & $22^{\circ} 26^{\prime} 84^{\prime \prime} \mathrm{N}$ y $84^{\circ} 31^{\prime} 19^{\prime \prime} \mathrm{W}$ & arrecife frontal \\
\hline
\end{tabular}

especie dominante fue Briareum asbestinum (Pallas, 1766), y Pseudopterogorgia bipinnata (Verrill, 1864) lo hizo para la localidad de Sancho Pardo. En la cresta arrecifal, Gorgonia ventalina Linnaeus, 1758 y Plexaura homomalla (Esper, 1792) fueron las que predominaron (Cuadro 2).

En la zona del arrecife frontal, los valores de densidad media $\left(\right.$ col. $\left.{ }^{*} \mathrm{~m}^{-2}\right)( \pm$ error estándar) fluctuaron entre $2.4 \pm 0.7$ en $\operatorname{los} 15 \mathrm{~m}$ de profundidad en Francisco Padre y el valor máximo de $11.0 \pm 3.7$ fue encontrado en los $10 \mathrm{~m}$ de La Tabla. En la zona trasera, estos valores fueron $6.0 \pm 2.4$ en Sancho Pardo $(5 \mathrm{~m}), 6.5 \pm 2.2$ en La Tabla $(5 \mathrm{~m})$ y en la cresta de La Tabla fueron $4.4 \pm 1.3$. Las diferencias más acentuadas en relación con la densidad se encontraron entre las localidades a 10 y $15 \mathrm{~m}$ de La Tabla y La Zorrita, con respecto a las mismas profundidades en Sancho Pardo, y los $15 \mathrm{~m}$ de El Pinto y Francisco Padre, que tuvieron la menor densidad (Fig. 2).
Las zonas del arrecife frontal presentaron un ITH bajo, que se incrementa al disminuir la profundidad. Según los valores de este indicador, en la zona trasera se infiere que la intensidad del oleaje es baja, mientras que esta se incrementa en la cresta (Cuadro 3).

El 79\% de las localidades mostraron un estado ambiental inferido de favorable a muy favorable, solo en Sancho Pardo este fue severo. E1 64\% de las localidades fue de ambientes constantes o casi constantes, mientras que el $36 \%$ restante fue no pronosticable (Cuadro 3).

\section{DISCUSIÓN}

Según Varona y Varela (2005), se han registrado para Cuba 77 especies de octocorales pertenecientes a 11 familias, de las cuales la familia Plexauridae posee el mayor número de especies, con 31. En los Bajos de Sancho Pardo, arrecife de Los 
Cuadro 2. Abundancia (\%) de octocorales en las localidades estudiadas de los Bajos de Sancho Pardo, arrecife de Los Colorados. SP = Sancho Pardo, FP = Francisco Padre, LT $=$ La Tabla, $\mathrm{LZ}=\mathrm{La}$ Zorrita y EP $=$ El Pinto

Table 2. Octocorals abundance (\%) in the study area of the Bajos de Sancho Pardo, in Los Colorados reef. SP $=$ Sancho Pardo, FP $=$ Francisco Padre, LT $=$ La Tabla, LZ = La Zorrita and $\mathrm{EP}=\mathrm{El}$ Pinto

\begin{tabular}{|c|c|c|c|c|c|c|c|c|c|c|c|c|c|c|}
\hline Especies/Localidad & SP & SP & SP & FP & FP & LT & LT & LT & LT & $\mathbf{L Z}$ & $\mathbf{L Z}$ & $\mathbf{L Z}$ & EP & EP \\
\hline Briareum asbestinum & 1 & 1 & 31 & 4 & 2 & 2 & 2 & 4 & 34 & 0 & 0 & 0 & 1 & 0 \\
\hline $\begin{array}{l}\text { Erythropodium } \\
\text { caribaeorum }\end{array}$ & 0 & 0 & 1 & 3 & 0 & 0 & 0 & 1 & 11 & 0 & 0 & 0 & 0 & 0 \\
\hline Pseudoplexaura porosa & 0 & 0 & 0 & 0 & 1 & 0 & 2 & 14 & 4 & 1 & 0 & 1 & 0 & 0 \\
\hline $\begin{array}{l}\text { Pseudoplexaura } \\
\text { flagellosa }\end{array}$ & 0 & 1 & 1 & 10 & 3 & 3 & 2 & 2 & 5 & 1 & 2 & 2 & 2 & 0 \\
\hline Pseudoplexaura crucis & 0 & 0 & 1 & 0 & 1 & 0 & 1 & 2 & 3 & 0 & 1 & 1 & 0 & 0 \\
\hline Muriceopsis flavida & 2 & 2 & 5 & 1 & 1 & 7 & 3 & 0 & 0 & 38 & 21 & 2 & 0 & 8 \\
\hline Plexaura homomalla & 0 & 0 & 2 & 0 & 0 & 0 & 0 & 21 & 10 & 0 & 1 & 2 & 0 & 0 \\
\hline Plexaura kuekenthali & 0 & 1 & 1 & 5 & 2 & 4 & 2 & 1 & 14 & 9 & 5 & 1 & 3 & 0 \\
\hline Eunicea flexиosa & 0 & 1 & 3 & 18 & 2 & 4 & 5 & 14 & 6 & 6 & 8 & 8 & 4 & 8 \\
\hline Eunicea mammosa & 1 & 0 & 2 & 1 & 1 & 2 & 2 & 7 & 3 & 4 & 6 & 25 & 1 & 12 \\
\hline Eunicea succinea & 0 & 0 & 0 & 1 & 3 & 1 & 2 & 0 & 1 & 2 & 0 & 0 & 2 & 1 \\
\hline Eunicea tourneforti & 0 & 0 & 1 & 4 & 2 & 2 & 2 & 8 & 2 & 5 & 4 & 8 & 1 & 6 \\
\hline Eunicea palmeri & 0 & 0 & 1 & 0 & 0 & 0 & 0 & 0 & 0 & 0 & 0 & 0 & 0 & 0 \\
\hline Eunicea clavigera & 0 & 0 & 0 & 1 & 0 & 0 & 0 & 0 & 0 & 1 & 1 & 0 & 0 & 0 \\
\hline $\begin{array}{l}\text { Eunicea calyculata } \mathrm{f} \text {. } \\
\text { coronata }\end{array}$ & 0 & 0 & 0 & 0 & 0 & 0 & 0 & 0 & 0 & 0 & 0 & 0 & 1 & 0 \\
\hline $\begin{array}{l}\text { Eunicea calyculata } \mathrm{f} . \\
\text { typica }\end{array}$ & 0 & 0 & 0 & 0 & 0 & 1 & 0 & 0 & 0 & 2 & 2 & 1 & 2 & 0 \\
\hline Plexaurella nutans & 0 & 0 & 0 & 1 & 1 & 1 & 1 & 0 & 0 & 0 & 1 & 0 & 0 & 0 \\
\hline Plexaurella dichotoma & 0 & 0 & 2 & 0 & 5 & 0 & 2 & 2 & 3 & 5 & 6 & 9 & 3 & 12 \\
\hline Plexaurella grisea & 0 & 0 & 1 & 0 & 1 & 0 & 0 & 0 & 1 & 0 & 1 & 1 & 1 & 1 \\
\hline Muricea pinnata & 0 & 0 & 0 & 0 & 1 & 0 & 0 & 1 & 0 & 1 & 3 & 5 & 0 & 4 \\
\hline Muricea muricata & 0 & 0 & 0 & 0 & 0 & 0 & 0 & 0 & 0 & 0 & 0 & 2 & 0 & 0 \\
\hline Muricea elongata & 0 & 0 & 0 & 0 & 0 & 0 & 0 & 1 & 0 & 1 & 0 & 2 & 0 & 0 \\
\hline Muricea atlantica & 0 & 0 & 1 & 0 & 0 & 0 & 1 & 0 & 0 & 0 & 0 & 1 & 0 & 1 \\
\hline Gorgonia ventalina & 4 & 10 & 8 & 1 & 1 & 6 & 7 & 21 & 3 & 3 & 9 & 4 & 3 & 3 \\
\hline Gorgonia mariae & 0 & 3 & 1 & 1 & 3 & 2 & 1 & 0 & 0 & 2 & 1 & 0 & 1 & 0 \\
\hline Pterogorgia citrina & 0 & 0 & 0 & 0 & 0 & 0 & 0 & 0 & 0 & 1 & 1 & 11 & 1 & 2 \\
\hline $\begin{array}{l}\text { Pterogorgia } \\
\text { guadalupensis }\end{array}$ & 0 & 0 & 0 & 0 & 0 & 0 & 0 & 0 & 0 & 0 & 0 & 0 & 1 & 0 \\
\hline Pterogorgia anceps & 0 & 0 & 0 & 0 & 0 & 0 & 0 & 0 & 0 & 0 & 0 & 0 & 7 & 9 \\
\hline $\begin{array}{l}\text { Pseudopterogorgia } \\
\text { americana }\end{array}$ & 16 & 11 & 1 & 19 & 35 & 6 & 17 & 2 & 1 & 6 & 17 & 11 & 46 & 27 \\
\hline $\begin{array}{l}\text { Pseudopterogorgia } \\
\text { elisabethae }\end{array}$ & 59 & 48 & 4 & 25 & 31 & 35 & 34 & 0 & 0 & 0 & 0 & 0 & 2 & 0 \\
\hline $\begin{array}{l}\text { Pseudopterogorgia } \\
\text { bipinnata }\end{array}$ & 17 & 21 & 35 & 0 & 1 & 15 & 5 & 0 & 1 & 2 & 2 & 2 & 2 & 0 \\
\hline $\begin{array}{l}\text { Pseudopterogorgia } \\
\text { acerosa }\end{array}$ & 1 & 2 & 0 & 1 & 4 & 5 & 10 & 0 & 0 & 10 & 9 & 2 & 16 & 6 \\
\hline $\begin{array}{l}\text { Pseudopterogorgia } \\
\text { rigida }\end{array}$ & 0 & 0 & 0 & 0 & 0 & 0 & 0 & 0 & 1 & 0 & 0 & 0 & 0 & 0 \\
\hline Eunicia laciniata & 0 & 0 & 0 & 1 & 0 & 1 & 1 & 0 & 0 & 1 & 1 & 0 & 0 & 0 \\
\hline Profundidad (m) & 15 & 10 & 5 & 15 & 10 & 15 & 10 & 1 & 5 & 15 & 10 & 5 & 15 & 10 \\
\hline
\end{tabular}




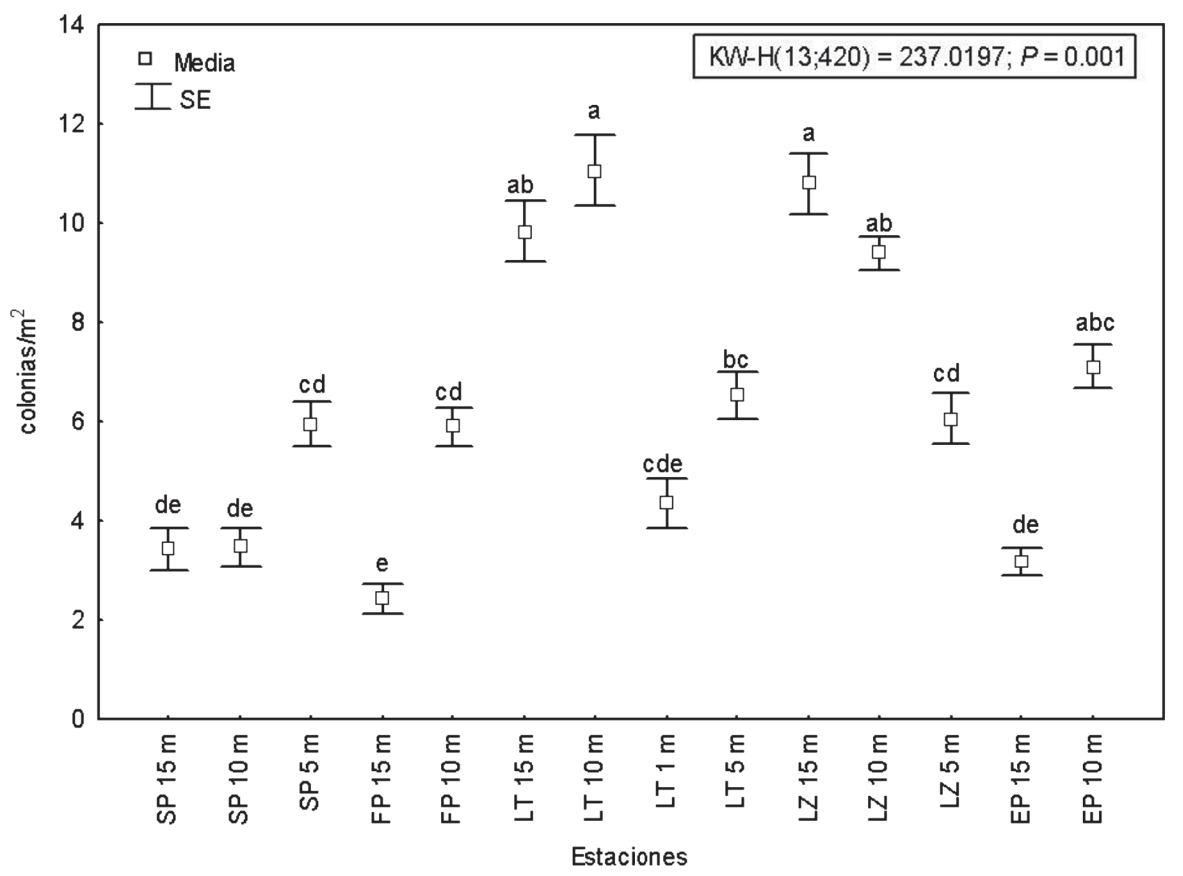

Fig. 2. Distribución de los valores de densidad obtenidos para la comunidad de octocorales de cada sitio estudiado. SE (error estándar). Las letras diferentes indican diferencias significativas. $\mathrm{SP}=$ Sancho Pardo, FP $=$ Francisco Padre, $\mathrm{LT}=\mathrm{La}$ Tabla, $\mathrm{LZ}=$ La Zorrita y EP $=$ El Pinto

Fig. 2. Distribution of density values obtained for the octocoral community of each studied site. $\mathrm{SE}$ (standard error). Different letters indicate significant differences. $\mathrm{SP}=$ Sancho Pardo, FP = Francisco Padre, $\mathrm{LT}=\mathrm{La}$ Tabla, $\mathrm{LZ}=\mathrm{La}$ Zorrita and $\mathrm{EP}=\mathrm{El}$ Pinto

Cuadro 3. Índices ecológicos e inferencias de comportamiento ambiental en cada estación en los Bajos de Sancho Pardo. N: número de colonias, S: número de especies, H': índice de heterogeneidad de Shannon, J': índice de equitatividad de Pielou, ITH: Índice de Tensión Hidrodinámica Table 3. Indexes of diversity and environmental inference in each station research in the Bajos de Sancho Pardo. N: Individuals number, S: species number, H': Shannon's diverity index, J': Pielou's eveness index, ITH: Hydrodynamic stress index

\begin{tabular}{lllllll}
\hline Estaciones & $\mathbf{N}$ & $\mathbf{S}$ & $\mathbf{H}^{\prime}$ & $\mathbf{J}^{\prime}$ & Comportamiento ambiental inferido & ITH (\%) \\
\hline SP $(15 \mathrm{~m})$ & 103 & 8 & 1.24 & 0.59 & severo y no pronosticable & 5 \\
SP $(10 \mathrm{~m})$ & 105 & 11 & 1.58 & 0.66 & severo y no pronosticable & 10 \\
SP $(5 \mathrm{~m})$ & 179 & 19 & 1.91 & 0.65 & severo y no pronosticable & 16 \\
FP $(15 \mathrm{~m})$ & 73 & 17 & 2.24 & 0.79 & favorable casi constante & 25 \\
FP $(10 \mathrm{~m})$ & 177 & 20 & 2.00 & 0.67 & favorable y no pronosticable & 11 \\
LT $(15 \mathrm{~m})$ & 295 & 22 & 2.30 & 0.75 & favorable casi constante & 16 \\
LT $(10 \mathrm{~m})$ & 331 & 23 & 2.30 & 0.73 & favorable casi constante & 18 \\
LT $(1 \mathrm{~m})$ & 131 & 15 & 2.17 & 0.80 & favorable y constante & 53 \\
LT $(5 \mathrm{~m})$ & 196 & 17 & 2.18 & 0.77 & favorable casi constante & 16 \\
EZ $(15 \mathrm{~m})$ & 324 & 22 & 2.28 & 0.74 & favorable casi constante & 26 \\
EZ $(10 \mathrm{~m})$ & 282 & 23 & 2.56 & 0.82 & muy favorable y constante & 35 \\
EZ $(5 \mathrm{~m})$ & 182 & 21 & 2.52 & 0.83 & muy favorable y constante & 68 \\
EP $(15 \mathrm{~m})$ & 96 & 20 & 2.05 & 0.68 & favorable y no pronosticable & 23 \\
EP $(10 \mathrm{~m})$ & 213 & 18 & 2.35 & 0.81 & favorable y constante & 51
\end{tabular}


Colorados, se identificó el $44 \%$ de las especies de octocorales reportadas por estos autores para Cuba. Además, la familia Plexauridae fue la más diversa con el $65 \%$ de las especies halladas, lo que coincide también con lo encontrado por Olivera $e t$ al. (2010), mientras que la familia Gorgoniidae fue la más abundante con el 51\% del total de los ejemplares contados.

La distribución, la abundancia, la forma y el tamaño de los Alcyonarios en diversos hábitats están influenciados por factores como: la iluminación, el régimen hidrodinámico, la presencia de sustrato de fijación y la sedimentación (Bayer, 1961; Botero, 1987). Ello se refleja en las diferencias encontradas para la densidad y la diversidad entre las localidades de estudio, donde coinciden la zona ecológica y la profundidad, por ejemplo, entre Francisco Padre y La Tabla en los $15 \mathrm{~m}$.

Según Alcolado (1981), el ITH es más alto en las localidades de menor profundidad. En la cresta arrecifal de La Tabla predominaron Plexaura homomalla, G. ventalina y Eunicea flexuosa (Lamouroux, 1821), las cuales representan el $56 \%$ de las especies encontradas. Caballero et al. (2006) obtuvieron los indicadores de ITH más elevados en la cresta del arrecife del Rincón de Guanabo, y entre las especies que dominaban este biotopo estaba $G$. ventalina.

En la cresta de La Tabla, el ambiente inferido fue favorable y constante, lo que contrasta con los moderados valores de tensión hidrodinámica aquí hallados. La influencia inferida para el oleaje está dada principalmente por la presencia de G. ventalina y E. flexuosa, ya que según Alcolado (1981), P. homomalla está catalogada como una especie con resistencia moderada al batimiento y no se utiliza para valorar el ITH. Además, De la Guardia et al. (2006) hallaron en arrecifes de la zona de buceo de Cayo Levisa, archipiélago de Los Colorados, que en la cresta la densidad de gorgonias disminuye en comparación con los biotopos de veril, macizos y arrecifes de parches. Esto coincide con lo encontrado en este estudio, donde los valores de densidad se pueden catalogar como bajos si se comparan con el arrecife frontal y la zona trasera de esa misma localidad (La Tabla).

En La Tabla, $P$. homomalla fue una de las especies más comunes en la zona trasera, donde la energía del oleaje es baja, lo que coincide con lo reportado por Alcolado et al. (2003). Las zonas traseras de Sancho Pardo y La Tabla están compuestas por cabezos, que dan mayor complejidad estructural a estos lugares y contribuyen a que la intensidad del oleaje sea menor. Aquí dominaron B. asbestinum, seguida de $P$. bipinnata, lo que concuerda con lo hallado por Varona et al. (2004), que encontraron que estas dos especies eran las más abundantes en la terraza somera. Alcolado et al. (2003) sugieren que $B$. asbestinum es una especie que abunda en la zona trasera del arrecife, e igualmente reportan en esta zona ecológica a Eunicea mammosa Lamouroux, 1816, E. flexuosa y P. dichotoma (Esper, 1791), lo cual coincide con nuestros resultados.

De forma general, el ambiente es favorable para el desarrollo de la comunidad de octocorales en el arrecife de los Bajos de Sancho Pardo, sin embargo, este fue severo en las estaciones ubicadas hacia el oeste. De la Guardia y González-Sansón (1997a) sugieren que el incremento de la profundidad, la pendiente de la pared y la mayor complejidad estructural del sustrato representan un ambiente más constante y favorable para el desarrollo de las comunidades de gorgonias. 
Las zonas de mayor diversidad de gorgonias coinciden con ser las más influenciadas por el mar abierto, donde hay un incremento de la incidencia del oleaje y el sustrato no está colonizado principalmente por corales y, por lo general, dominan $P$. americana y E. flexuosa (De la Guardia y González-Sansón, 1997a). Esto se cumple en todas las localidades estudiadas en el arrecife frontal, menos para Sancho Pardo.

El comportamiento ambiental inferido a partir de los índices de diversidad mostró ser no pronosticable en Sancho Pardo y Francisco Padre. Ello podría deberse a que la protección durante eventos meteorológicos extremos es menos efectiva en estos puntos del arrecife. Un comportamiento similar fue reportado por Hernández y Alcolado (2007). Además, aquí el arrecife frontal estaba cubierto por macroalgas, las cuales colonizaban una gran parte del sustrato. En relación con lo anterior, Gotelli (1988) y Chadwick y Morrow (2011) plantean que la presencia de macroalgas inhibe el reclutamiento en los octocorales y provoca la reducción de la supervivencia de los reclutas que logran fijarse al sustrato. Al respecto, Richmond (1997) considera que los corales adultos pueden sobrevivir en áreas donde la reproducción está fallando y las larvas son incapaces de fijarse.

En el arrecife frontal, $P$. americana, $P$. elisabethae y Muriceopsis flavida (Lamarck, 1815) fueron las especies que dominaron, y la primera de ellas estuvo en todas las localidades. P. americana es considerada por Alcolado (1990) como una especie euritópica. Esta especie es la dominante en el veril del Rincón de Guanabo (Caballero et al. 2006), en la terraza somera $(5 \mathrm{~m})$ del Área Protegida Centro-Oeste Cayo Coco (Hernández y Alcolado, 2007) y en la explanada frontal (De la Guardia y González-Sansón, 1997b).
Olivera et al. (2010) plantean que $E$. flexuosa, E. mammosa, Eunicea tourneforti Milne Edwards \& Haime, 1857, P. dichotoma y Pterogorgia citrina (Esper, 1792) son características de ambientes turbulentos. En La Zorrita, en los cinco metros de profundidad, se encontró que las especies antes citadas dominaban este hábitat, lo que influyó en el valor del ITH obtenido.

La morfología de las gorgonias previene la acumulación de sedimentos en la superficie de las colonias y la sedimentación es uno de los parámetros que afectan el reclutamiento de las larvas de coral (Rogers, 1990). Herrera y Alcolado (1983) plantean que $P$. dichotoma, Pterogorgia guadalupensis, Pterogorgia anceps (Pallas, 1766), $P$. citrina, $P$. americana y $P$. acerosa $(\mathrm{Pa}-$ llas, 1766) son especies resistentes a la sedimentación. La abundancia de estas especies en El Pinto permitió inferir que dicho factor juega un papel fundamental en la estructura y composición de la comunidad de octocorales que aquí se desarrolla.

González-Díaz et al. (2010) establecieron la línea base de la composición de las comunidades de corales, esponjas y gorgonias en el arrecife frontal de Los Colorados en los sitios Francisco Padre, La Tabla, La Zorrita y El Pinto. Aunque el enfoque de este trabajo fue principalmente hacia la comunidad de corales, se muestra que los valores de la densidad de gorgonias $\left(\mathrm{col} .{ }^{*} \mathrm{~m}^{-2}\right)$ oscilaron entre 5.6 (Francisco Padre) y 8.1 (La Zorrita) en el biotopo del veril y 2.5 (Francisco Padre) y 8.2 (La Zorrita) en el biotopo de macizos y canales. En esta investigación también se registró una alta densidad de octocorales en el arrecife frontal de La Zorrita y los valores más bajos en Francisco Padre.

En general, el ITH mostró una tendencia a ser bajo, con la excepción de aquellos lugares donde disminuía la profundidad y 
aumentaba la exposición al oleaje (5 $\mathrm{m}$ de La Zorrita y La Tabla $1 \mathrm{~m}$ ). Esto se manifiesta en las zonas del arrecife frontal, con la presencia de especies poco tolerantes al batimiento en los $15 \mathrm{~m}$, como $P$. bipinnata y $P$. elisabethae, las cuales van siendo sustituidas por especies de tolerancia moderada, como P. americana y Pseudopterogorgia acerosa, a alta, como G. ventalina, E. flexuosa, E. mammosa y algunas Pterogorgia sp., lo que concuerda con lo descrito por Alcolado (1981) y Olivera et al. (2010).

El arrecife de los Bajos de Sancho Pardo ha sufrido el impacto de eventos extremos como los huracanes Helene en el 2000, Isidore y Lili en el 2002, Bonnie e Iván en el 2004, Arlene en el 2005, así como Gustav y Ike en el 2008. Además, en este impactan los frentes fríos de la temporada invernal, que incrementan la intensidad del oleaje. Al respecto, los efectos de estos fenómenos ambientales no pueden ser medidos con claridad a partir de los datos obtenidos para estas comunidades de octocorales. Sin embargo, Goffredo y Lasker (2006), al analizar el crecimiento modular de la especie Pseudopterogorgia elisabethae, hallaron que esta mostró una etapa de reclutamiento exitoso después del paso del huracán Andrew en 1992.

Al respecto, Yoshioka y Yoshioka (1989), al observar el carácter impredecible de las consecuencias de los efectos del huracán David sobre las especies de octocorales, cuestionaron la susceptibilidad de estos al oleaje. Connell (1978) plantea en su hipótesis que la incidencia de eventos meteorológicos fuertes (huracanes y tormentas severas), que ocurran con frecuencias intermedias, puede favorecer que la diversidad de especies sea alta, permitiendo que nuevos individuos colonicen el sustrato que queda libre, y así se minimiza el efecto de la exclusión competitiva.

\section{CONCLUSIONES}

Las comunidades de octocorales de los Bajos de Sancho Pardo mostraron diferencias significativas en su estructura para algunos de los sitios estudiados, tanto entre localidades de un mismo sitio a diferentes profundidades como entre iguales localidades de sitios diferentes. Condiciones ecológicas variables, como la profundidad, la resuspensión de sedimentos o la abrasión del oleaje pueden ser algunos de los factores determinantes en esta distribución.

El comportamiento ambiental inferido a partir de los índices de diversidad mostró que la mayoría de las localidades presentaron condiciones favorables para el desarrollo de las comunidades de gorgonáceos, a pesar de encontrarse este archipiélago frecuentemente impactado por fenómenos atmosféricos severos como huracanes de gran intensidad y frentes fríos, lo que supone una fuerte resiliencia del grupo ante fenómenos de esta naturaleza.

\section{BIBLIOGRAFÍA}

Alcolado, P. M. (1981). Zonación de los gorgonáceos someros de Cuba y su posible uso como indicadores comparativos de tensión hidrodinámica sobre los organismos del bentos. Inf. Cient. Téc. Inst. Oceanol., 187, 1-43.

Alcolado, P. M. (1990). Breve comentario acerca de las comunidades de gorgonáceos de la macrolaguna del Golfo de Batabanó. En P. M. Alcolado (Ed.), El bentos de la macrolaguna del Golfo de Batabanó (pp. 34-36). La Habana, Cuba: Editorial Academia.

Alcolado, P. M., Claro, R., MartínezDaranas, B., Menéndez, G., García, P. \& Sosa, M. (2003). The Cuban Coral 
Reefs. En J. Cortés (Ed.), Latin American Coral Reefs (pp. 53-76). San José, Costa Rica: Elsevier.

Alcolado, P. M., García-Parrado, P. \& Hernández-Muñoz, D. (2008). Estructura y composición de las comunidades de gorgonias de los arrecifes del Archipiélago Sabana-Camagüey, Cuba: conectividad y factores determinantes. Bol. Invest. Mar. Cost., 37(1), 11-29.

Bayer, F. M. (1961). The shallow water Octocorallia of the West Indian Region. A manual for marine biologists. The Hague, Netherlands: Martinus Nijhoff.

Botero, L. (1987). Zonación de octocorales en el área de Santa Marta y Parque Nacional Tayrona, Costa Caribe colombiana. An. Inst. Invest. Mar., 17, 61-80.

Caballero, H., Rosales, D. \& Alcalá, A. (2006). Estudio diagnóstico del arrecife coralino del Rincón de Guanabo, Ciudad de La Habana, Cuba. 1. Corales, Gorgonáceos y Esponjas. Rev. Invest. Mar., 27(1), 49-59.

Cadena, N. J. \& Sánchez, J. A. (2010). Colony growth in the harvested octocoral Pseudopterogorgia acerosa in a Caribbean coral reef. Mar. Ecol., 31(4), 566-573.

Chadwick, N. E. \& Morrow, K. M. (2011). Competition among sessile organisms on coral reef. En Z. Dubinsky \& N. Stambler (Eds.), Coral Reefs: an ecosystem in transition (pp. 347-371). Dordrecht, Netherlands: Springer.

Connell, H. J. (1978). Diversity in tropical rain forests and coral reefs. Science, 199(4335), 1302-1310.

De la Guardia, E. \& González-Sansón, G. (1997a). Asociaciones de esponjas, gorgonias y corales de un arrecife en la costa noroccidental de Cuba. III:
Variación espacial de la diversidad. Rev. Invest. Mar., 18(3), 216-222.

De la Guardia, E. \& González-Sansón, G. (1997b). Asociaciones de esponjas, gorgonias y corales de un arrecife en la costa noroccidental de Cuba. I: Distribución espacial de biotopos. Rev. Invest. Mar., 18(3), 198-213.

De la Guardia, E., González-Díaz, P., Valdivia, A. \& González, O. (2006). Estructura y salud de la comunidad de corales en arrecifes de la zona de buceo de Cayo Levisa, Archipiélago de Los Colorados, Cuba. Rev. Invest. Mar., 27(3), 197-208.

Drohan, A. F., Thoney, D. A. \& Baker, A. C. (2005). Synergistic effect of high temperature and ultraviolet-B radiation on the gorgonian Eunicea tournefourti (Octocorallia: Alcyonacea: Plexauridae). Bull. Mar. Sci., 77(2), 257-266.

Goffredo, S. \& Lasker, H. R. (2006). Modular growth of a gorgonian coral can generate predictable patterns of colony growth. J. Exp. Mar. Biol. Ecol., 336, 221-229.

González-Díaz, P., González-Sansón, G., Álvarez-Fernández, S. \& Perera-Pérez, O. (2010). High spatial variability of coral, sponges and gorgonian assemblages in a well preserved reef. Rev. Biol. Trop., 58(2), 621-634.

González-Sansón, G., Aguilar, C., Hernández, I., Cabrera, Y. \& Curry, A. (2009). The influence of habitat and fishing on reef fish assemblages in Cuba. Gulf Carib. Res., 21, 13-21.

Gotelli, N. J. (1988). Determinants of recruitment, juvenile growth, and spatial distribution of a shallow-water gorgonian. Ecology, 69(1),157-166.

Hernández, L. F. \& Alcolado, P. M. (2007). Estructura de la comunidad de octocorales de una zona propuesta como área 
protegida en Cayo Coco, Cuba. Rev. Invest. Mar., 28(3), 209-216.

Hernández-Muñoz, D., Alcolado, P. M. \& Hernández-González, M. (2008). Efecto de un emisario submarino de residuos urbanos sobre comunidades de octocorales (Octocorallia: Alcyonacea) en Cuba. Rev. Biol. Trop., 56(1), 65-75.

Herrera, A. \& Alcolado, P. M. (1983). Efectos de la contaminación sobre comunidades de gorgonáceos al oeste de la Bahía de La Habana. Cien. Biol., 10, 69-95.

Keller, B. D., Gleason, D. F., McLeod, E., Woodley, C. M., Airamé, S., Causey, B. D., Friedlander, A. M., Grober-Dunsmore, R., Johnson, J. E., Miller, S. L. \& Steneck, R. S. (2009). Climate change, coral reef ecosystems, and management options for marine protected areas. Environ. $\mathrm{Ma}$ nage., 44, 1069-1088.

Lasker, H. R. (2003). Zooxanthella densities within a Caribbean octocoral during bleaching and non-bleaching years. Coral Reef, 22(1), 23-26.

Olivera, Y. E., Hernández-Fernández, L. \& Jover, A. C. (2010). Estructura y daños en las comunidades de octocorales (Octocorallia: Alcyonacea) de la Reserva Ecológica Siboney-Juticí, Santiago de Cuba, Cuba. Rev. Biol. Trop., 58(4), 1211-1221.

Pielou, E. C. (1966). Shannon's formula as a measurement of specific diversity: its use and mesure. Am. Nat., 100(914), 463-465.

Richmond, R. H. (1997). Reproduction and recruitment in corals: critical links in the persistence of reef. En C. Birkeland (Ed.), Life and Death of Coral Reefs (pp. 175-197). New York, EE. UU.: Chapman \& Hall.
Rogers, C. S. (1990). Responses of coral reefs and reef organisms to sedimentation. Mar. Ecol. Prog. Ser., 62, 185-202.

Sánchez, J. A. \& Wirshing, H. H. (2005). A Field Key to the Identification of Tropical Western Atlantic Zooxanthellate Octocorals (Octocorallia: Cnidaria). Caribb. J. Sci., 41(3), 508-522.

Schutte, V. G. W., Selig, E. R. \& Bruno, J. F. (2010). Regional spatio-temporal trends in Caribbean coral reef benthic communities. Mar. Ecol. Prog. Ser., 402, 115-122.

Shannon, C. E. \& Weaver, W. (1949). The mathematical theory of communication. Illinois, EE.UU.: University of Illinois Press, Urbana.

Varona, G., Caballero, H. \& De la Guardia, E. (2004). Estructura ecológica de las comunidades de octocorales en la costa oriental de Bahía de Cochinos, Cuba. Rev. Invest. Mar., 25(3), 209-218.

Varona, G. \& Varela, C. (2005). Primer hallazgo de Eunicea pinta Bayer y Deichmann 1958 (Cnidaria, Gorgonacea) en aguas cubanas. COCUYO, 15, 2-3.

Vega, A., Font, Y. \& Zayas, C. (2002). Composición de la fauna más representativa en el arrecife de coral costero de playa Corinthia, región nororiental de Cuba. Rev. Invest. Mar., 23(3), 167-172.

Weinberg, S. (1981). A comparison of Coral Reef Survey Methods. Contrib. Zool., 51, 199-218.

Yoshioka, P. M. \& Yoshioka, B. B. (1989). Effects of wave energy, topographic relief and sediment transport on the distribution of shallow watergorgonians of Puerto Rico. Coral Ree$f_{s}, 8,145-152$. 